\title{
Semantic Sensor Service Networks
}

\author{
Wei Wang, Payam Barnaghi, Gilbert Cassar, Frieder Ganz, Pirabakaran Navaratnam \\ Centre for Communication Systems Research \\ University of Surrey \\ Guildford, Surrey, United Kingdom \\ \{wei.wang, p.barnaghi, g.cassar, f.ganz, p.navaratnam\}@ surrey.ac.uk
}

\begin{abstract}
Sensors and sensor networks are fundamental instruments to acquire and communicate contextual information from the physical world. This information enables better understanding of the physical world for humans and supports creation of ambient intelligence for a wide range of applications in different domains such as smart cities, healthcare and intelligent transportation. To facilitate scalable and seamless access and management of the information obtained from large and heterogeneous sensor networks, we introduce the concept of Semantic Sensor Service Networks which brings the research on Semantic Sensor Network one-step further. The concept combines semantic modelling, knowledge management and service-oriented design to support sensor service access, discovery and composition. It defines a framework in which sensor services can collaborate and co-operate to support data integration from different sensor networks and service computing for complex business applications.
\end{abstract}

\section{INTRODUCTION}

There are increasing number of sensors that report data from our physical environment. Due to the large-scale, distributed and heterogeneous nature of the sensor networks, the research community has used semantic technologies for sensor and sensor data representation. A notable work in this line is the collaborative efforts from academia and industry towards the development of the Semantic Sensor Network Ontology (SSN) [1]. We anticipate that the next step should focus on unconstrained access to the data generated from the heterogeneous sensor networks, which is the key for applications that require context and situation awareness. The service oriented computing mechanisms provide standardised means for communication and integration among systems and provide potential to develop scalable and loosely coupled software systems. Representing sensor as a service in the cyber-world has been considered as a potential solution for efficient management of the sensor data. However, most of the sensors operate in the highly dynamic physical world and the exposed sensor services are not as reliable and stable as those well-engineered and maintained business services. This brings significant challenges to sensor service computing such as seamless service connectivity, discovery and dynamic

This work is supported by the European Union Seventh Framework Programme IoT.est project: Internet of Things Environment for Service Creation and Testing (http://ict-iotest.eu/iotest/), contract number: 257521. service composition with efficient adaptation and compensation mechanisms.

In this paper, we discuss our ongoing work on integrating the techniques from the semantic Web and service oriented computing to address these challenges. The rest of the paper is organised as follows. In Section 2, we review some of the existing works on semantic and service computing on sensor and sensor networks. In Section 3, we introduce the concept of semantic sensor service networks and discuss the challenging problems on applying service oriented computing for sensor data access and management. Section 4 outlines the future research issues and concludes the paper.

\section{RELATED WORK}

Much of the existing research on sensors and sensor networks has focused on developing standardised languages for sensor data representation and encoding, notably, the Sensor Web Enablement (SWE) initiative [2]. Recognising the heterogeneity of sensing devices, their data formats, and measurement procedures, The W3C Semantic Sensor Network Incubator group developed the SSN ontology for semantically describing sensors, observations/measurement, and sensor systems based on the Stimulus-SensorObservation design pattern [1]. As one of the most fundamental components of the "Internet of Things" (IoT), sensors and sensor networks provide real-world information and enable us to develop context-aware applications and services. What is important here is the unconstrained access to the sensor observation and measurement data.

Data access in (wireless) sensor networks can be implemented at device or network levels by using low-level programming languages and operating systems [3], but the heterogeneity of the devices and (sensor) networks makes data access across the networks a difficult task. Service oriented principles, which allow complex software systems to be decomposed into smaller sub-systems or services have been used to integrate the sensor data with enterprise services [4]. The idea of "sensing as a service" represents a scalable way to access the sensor data through standard service technologies and has received consensus from the 
community. The SemSense architecture proposed a real world data management approach by collecting, annotating and publishing data onto the Web based on the linked data principle, nevertheless, it does not support on-demand sensor data access [5].

Service oriented computing mechanisms have been proposed and integrated with semantic technologies to address the challenging issues related to heterogeneity and interoperability in sensor data access and management, for example, De et al described a semantic sensor service model for resources and devices (e.g., sensors) in the IoT [6]. Guinard et al proposed a service oriented architecture for the IoT and identified several essential requirements for computing with real-world services for enterprise applications [7]. They developed methods for service registration, discovery (with a simple service instance ranking mechanism) and on-demand provisioning according to those requirements. However, their work is intended to facilitate service developers and designers to dynamically discover services that still requires considerable human efforts; the lack of semantic descriptions also creates potential problems for large-scale real-world services. Furthermore, service composition with efficient adaptation and compensation mechanism were not discussed.

\section{Semantic SEnsor Service Network}

The notion of Semantic Sensor Service Network (See Figure 1) is introduced to facilitate seamless access and management of sensor observation and measurement data based on service oriented computing mechanisms and semantic technologies.

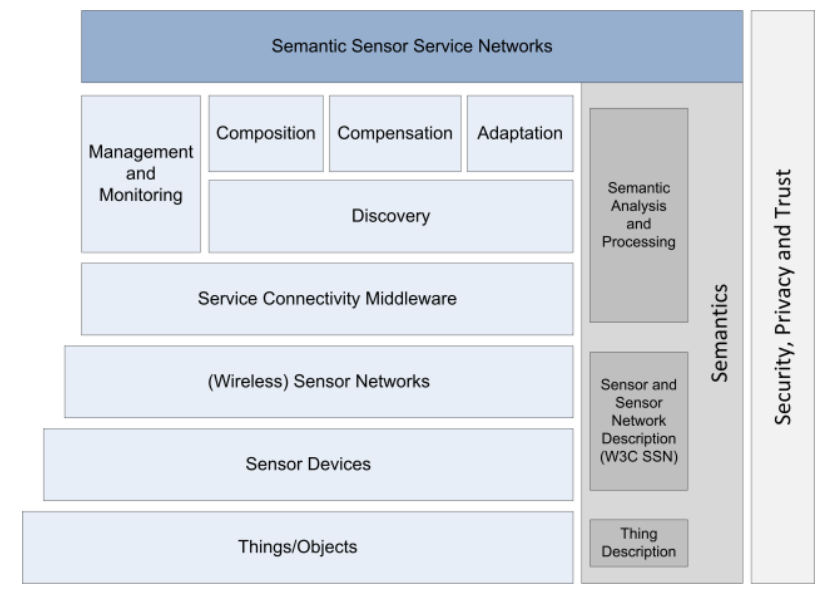

Figure 1. A layered model for the Semantic Sensor Service Network

The Semantic Sensor Service Network brings the research on Semantic Sensor Network one-step further by adding several service related layers on the top of the sensor and sensor networks (or more general, IoT). It defines a framework in which sensor services can collaborate and cooperate to support data access and integration from different sensor networks for various business services and applications that need the real-world information for intelligent decision making. With the semantic descriptions and service interfaces, it enables unconstrained access to large-scale and distributed sensor services across heterogeneous sensor networks. Another significant advantage is that sensor services can be potentially discovered and integrated with existing (high-level business) services and applications that need real-world data by using existing standard (Web) service computing techniques. However, sensor services manifest distinctive characteristics in several ways compared to relatively reliable and carefully maintained enterprise services: sensors exposing services usually operate in dynamic environments; they may be deployed in different platforms and communicate using different protocols; they are often mobile and mostly capability-constrained. These characteristics highlight the importance of sensor service connectivity, discovery and composition with effective adaptation and compensation mechanisms. In the following, we describe the semantic sensor service model, the gateway component that provides service connectivity as well as sensor service discovery and composition issues. Sensor observation and measurement data processing and abstraction to create ambient intelligence is another important research topic, however, they are out of the scope of this paper and will not be discussed here.

\section{A. Semantic Description for Sensor Services}

The evolution of large-scale sensor service networks requires a holistic understanding of the dynamics, capabilities, and interdependencies of their enabling technologies. Providing unambiguous descriptions for sensors and observation/measurement data becomes one of the most important tasks for sensor service networks, considering the scale, distributed, heterogeneous and dynamic nature of the sensors. Semantic Web technologies have been successfully used to model and describe sensor devices, for example, the W3C Semantic Sensor Network Incubator group has developed the Semantic Sensor Network ontology [1] to describe sensor devices, their physical and operational properties. The ontology also provides abstract concepts related to observation and measurement data models and platform descriptions. However, using semantic descriptions (especially ontology languages $\mathrm{RDF}^{1}$ or $\mathrm{OWL}^{2}$ ) is not that straightforward in the sensor networks for publication and exchange of data: the heavy payload of the XML serialisation of these representations is unsuitable for constrained environments (e.g., sensor networks). Recently there have been works that proposed to use lightweight semantics for knowledge representation in these environments; e.g., Binary $\mathrm{RDF}^{3}$ proposes a compressed representation scheme for RDF data; SA-REST ${ }^{4}$ defines a lightweight framework for describing services. Apart from the lightweight representation schemes that provide a reasonable balance between expressiveness and computation complexity, the service oriented technologies on the Web and subsequently for the sensor networks are moving towards RESTful solutions.

\section{B. Sensor Service Connectivity}

To enable seamless connectivity in a service-oriented environment sensors have to implement standard service

\footnotetext{
${ }^{1}$ http://www.w3.org/RDF/

${ }^{2}$ http://www.w3.org/2004/OWL/

${ }^{3} \mathrm{http}: / /$ www.w3.org/Submission/2011/03/

${ }^{4}$ http://www.w3.org/Submission/SA-REST/
} 
interfaces and communication protocols. Most of the existing service standards are designed for enterprise services and Web APIs based on HTTP, which in many cases cannot be applied to resource-constrained devices.

CoAp [8] is one of the recent standardisation efforts to introduce HTTP service based solutions for the constrained environments such as IoT and sensor networks. It is based on 6LowPan ${ }^{5}$, which is a customised IP protocol to enable HTTP based services (similar to RESTful services) on low-level devices. However, using CoAp and 6LowPan to provide services for the Internet-based service oriented architecture running on the IP protocol requires intermediate gateways or proxies to translate between the customised protocols and the Internet protocols. Besides running on intermediate nodes, services can also run directly on sensor nodes. Delicato et al [9] proposed an architecture in which the service is provided by the sensor itself via the WS-* standards that are usually used in business environments. This solution requires nodes with high processing capabilities as the standards relies heavily on XML processing.

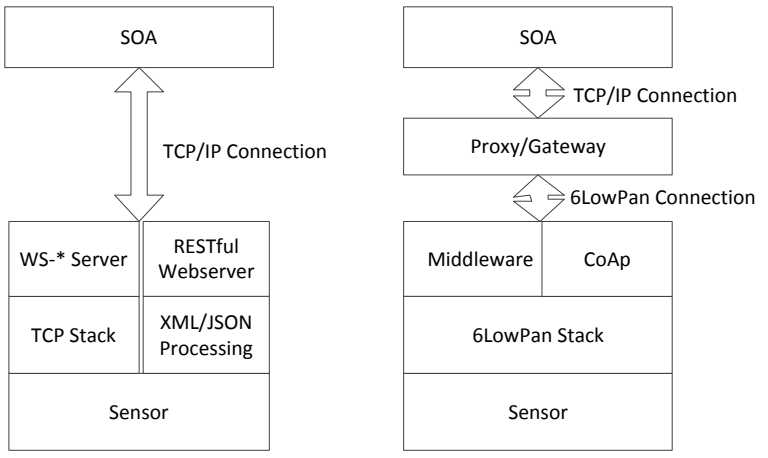

Figure 2. Two ways to access sensor services: Direct vs. Intermediate

This leads to two potential ways to render a sensor as a service. The first is to run services directly on a sensor which has sufficient processing resources (Figure 2, left). Another way is to run sensor services on intermediate nodes (such as gateways) which act as protocol translators between the constrained environments and the Internet (Figure 2, right). The advantages and limitations of these two sensor service connectivity methods are summarised in Table 1.

TABLE I. TABLE TYPE STYLES

\begin{tabular}{|c|c|}
\hline $\begin{array}{l}\text { Service rendered on } \\
\text { Device }\end{array}$ & $\begin{array}{c}\text { Service rendered on } \\
\text { intermediate node accessing } \\
\text { sensor node }\end{array}$ \\
\hline + Direct Sensor Access & $\begin{array}{c}\text { +Better In-network } \\
\text { aggregation }\end{array}$ \\
\hline $\begin{array}{c}\text {-Only on strong sensor } \\
\text { nodes }\end{array}$ & + Possible Caching \\
\hline \multirow[t]{2}{*}{ +More Privacy Control } & $\begin{array}{c}+ \text { for really constrained } \\
\text { devices }\end{array}$ \\
\hline & $\begin{array}{c}\text {-Intermediate Processing } \\
\text { needed/less flexible topology } \\
\text { wise }\end{array}$ \\
\hline
\end{tabular}

\footnotetext{
${ }^{5}$ http://datatracker.ietf.org/wg/6lowpan/charter/
}

\section{Sensor Service Discovery}

Service discovery is fundamental for service oriented computing as other high-level service oriented concepts such as service composition, provisioning, and adaptation highly rely on the results of service discovery. To be useful in dynamic environments such as sensor networks, service discovery needs to implement automated matchmaking approaches, preferably based on both functional and nonfunctional attributes of services. Service discovery in resource-constrained environments is more challenging than discovery on the Internet where reliable service resources are abundant. Sensor nodes are limited in processing capabilities and energy (e.g., limited battery life); communication between sensor nodes and gateways is also error prone and in many cases unreliable; the changes of the surrounding environments also have significant impact on performance of the sensors. To efficiently discover sensor services in such the dynamic environments, a publishing scheme that keeps semantic service descriptions up-to-date in the service registries is needed. At the same time, the communication should use minimum service advertisement messages in order to reduce the energy consumption and communication overheads.

The semantic descriptions for sensor services contain all the functional and non-functional data required for efficiently finding the required services. This semantic data includes: input and output parameter types and how they change with time, logical expressions that describe the operations in the service, any transformations performed on the data, location information about the observation area of the service and how it changes over time. The descriptions should also contain information describing QoS (e.g., reliability,), communication and processing costs for the service during runtime. Service discovery can be performed by creating a semantic service request, based on any of the semantic data contained in the semantic sensor service descriptions, and using a matchmaking mechanism to find the services that best match the request. The results are then ranked in order of relevance to the request.

\section{Sensor Service Composition}

Static and manual (or even semi-automated) service composition planning is an unsuitable solution for service composition in sensor networks because of the dynamic environment and unreliability of sensor nodes. We need automatic and dynamic mechanisms that can compose services automatically or efficiently compensate for a service that becomes unavailable during runtime. Approaches to dynamic service composition take either a top-down or a bottom-up approach. A top-down approach starts from a service request and proceeds to look for components that can match that request until enough components are found to form a usable service composition. A bottom-up approach creates a database of possible service compositions before runtime. In order to achieve dynamic service composition that involves sensor services, we need a mechanism that is able to decompose a service request into simpler parts to make it easier to search for services that provide the functionalities of those different parts. 
We propose a decomposition method that can be used at runtime to repeatedly divide a service request into smaller subrequests. The process will be repeated until for each subrequest we find at least one atomic service that is able to meet the requirement of that sub-request. The atomic services can then be used to create a composite service that is capable of providing the requirements of the original request.

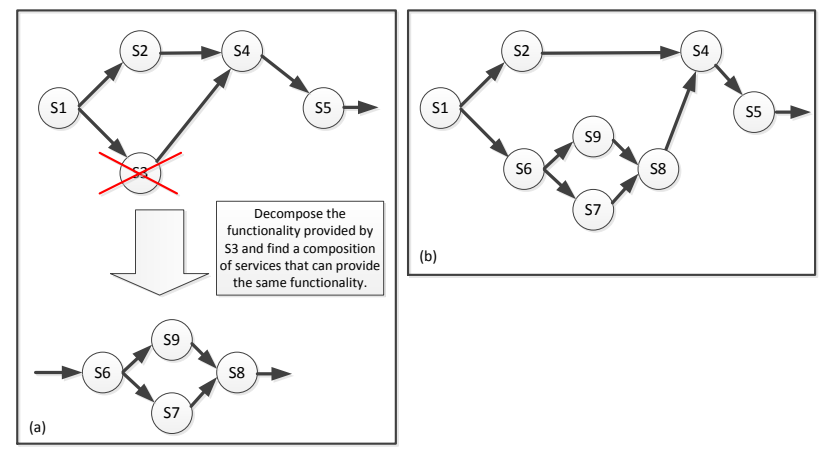

Figure 3. Illustration of service composition and compensation

In semantic sensor networks the service conditions might change frequently and sometimes a service might disappear for various reasons (e.g., low battery or network brokage). In cases where a service is not accessible anymore while it is being used in a service composition, a service compensation mechanism is needed to compensate for the loss of that service. The efficiency of service compensation at providing a seamless continuation of the service functionality is highly dependent on an updating scheme that notifies the compensation mechanism whenever a service from a composite service is unavailable. This ensures that the compensation mechanism has enough time to find a suitable replacement for that service. Service compensation works by creating a service request that corresponds to the service functionality that went missing and discovering a service that is capable of providing same or similar functionality. However, it might be the case that no such service exists. In such a case, the compensation mechanism should search for an atomic or composite service that can replace the missing service (See Figure 3, the functionality of service S3 is replaced with a composite service). This would involve decomposing the service request to smaller sub-requests automatically during runtime so that simpler services can be found for each sub-request and composed together to provide the required capabilities. In such situations, the required service functionality is decomposed into simpler requests and a mechanism similar to dynamic service composition is used. This mechanism keeps decomposing the required functionality until a number of service components that (when combined together) provide the required functionality are found.

\section{CONCLUSION AND FUTURE RESEARCH}

Sensor service connectivity, discovery and composition are some of the most prominent issues in semantic sensor service network. To enable seamless connectivity for the semantic sensor services, we propose two methods to access sensor services based on the service-oriented architecture: direct and intermediate access. While the direct access method uses the standard HTTP protocols for service communications, the intermediate access method is designed on the top of the
Constrained Application Protocol (CoAp) and 6LowPan for devices operating in constrained environments. A gateway that is able to translate between CoAp and the HTTP protocols has been designed to support communications between sensor services and existing high-level Web services.

Service discovery is an important functionality in the highly dynamic semantic sensor service networks and is fundamental for service composition, compensation and adaptation. To efficiently discover sensor services in real world environment, a publication scheme that keeps semantic service descriptions in registries (e.g., gateways) up-to-date and sends minimum service advertisement messages is used. For service composition we propose a method that can be used at runtime to repeatedly divide a service request into simpler sub-requests. The process will be repeated until for each subrequest we can find at least one atomic service that provides the requirements for that sub-request. When a sensor service becomes unavailable at runtime, an efficient compensation mechanism is needed in order to minimise service disruption time.

Our future work involves improving current service discovery methods, in particular, sensor service ranking, based on semantic reasoning and the linked data on the semantic Web. Another research issue is to develop intelligent compensation services based on approximate analysis of the data, networks and services of sensors for the situations where existing compensation methods fail to provide solutions.

\section{REFERENCES}

[1] M. Compton, P. Barnaghi, L. Bermudez, R. Garcia-Castro, O. Corcho, S. Cox, J. Graybeal, M. Hauswirth, C. Henson, A. Herzog, V. Huang, K. Janowicz, W. Kelsey, D. Le Phuoc, L. Lefort, M. Leggieri, H. Neuhaus, A. Nikolov, K. Page, A. Passant, A. Sheth, K. Taylor. "The SSN Ontology of the W3C Semantic Sensor Network Incubator Group", Journal of Web Semantics, 2012.

[2] M. Botts, G. Percivall, C. Reed, J. Davidson, OGC Sensor Web Enablement: Overview And High Level Architecture, OpenGIS White Paper OGC 07-165, Open Geospatial Consortium Inc., 2007.

[3] Oscar Corcho and Raúl García-Castro. 2010. Five challenges for the Semantic Sensor Web. Semant. web 1, 1,2 (April 2010), 121-125.

[4] Spiess, P. et al (2009), "SOA-Based Integration of the Internet of Things in Enterprise Services", In Proceedings of the 2009 IEEE International Conference on Web Services (ICWS '09). IEEE Computer Society, Washington, DC, USA, 968-975.

[5] Alexandra Moraru, Dunja Mladenic, Matevz Vucnik, Maria Porcius, Carolina Fortuna, and Mihael Mohorcic. 2011. Exposing real world information for the web of things. In Proceedings of the 8th International Workshop on Information Integration on the Web: in conjunction with WWW 2011 (IIWeb '11).

[6] S. De, T. Elsaleh, P. Barnaghi , S. Meissner, "An Internet of Things Platform for Real-World and Digital Objects", Journal of Scalable Computing: Practice and Experience, vol 13, no.1, 2012.

[7] D. Guinard, V. Trifa, S. Karnouskos, P. Spiess, D. Savio, "Interacting with the SOA-Based Internet of Things: Discovery, Query, Selection, and On-Demand Provisioning of Web Services," IEEE Transactions on Services Computing, pp. 223-235, July-September, 2010.

[8] Carsten Bormann, Angelo Paolo Castellani, and Zach Shelby. CoAP: An Application Protocol for Billions of Tiny Internet Nodes.. IEEE Internet Computing, (16)2:62-67, 2012.

[9] Delicato, F.C.; Pires, P.F.; Pinnez, L.; Fernando, L.; da Costa, L.F.R.; , "A flexible web service based architecture for wireless sensor networks," Distributed Computing Systems Workshops, 2003. Proceedings. 23rd International Conference on , vol., no., pp. 730- 735, 19-22 May 2003. 